\title{
Sofosbuvir: A Potential Treatment for Ebola
}

\author{
Sandra E. Reznik ${ }^{1,2}$, Amit K. Tiwari ${ }^{3}$ and Charles R. Ashby Jr. ${ }^{1 *}$ \\ 1 Department of Pharmaceutical Sciences, College of Pharmacy and Health Sciences, St. John's University, Queens, NY, \\ United States, ${ }^{2}$ Departments of Pathology and Obstetrics and Gynecology and Women's Health, Montefiore Medical \\ Center/Albert Einstein College of Medicine, Bronx, NY, United States, ${ }^{3}$ Department of Pharmacology and Experimental \\ Therapeutics, College of Pharmacy and Pharmaceutical Sciences, University of Toledo, Toledo, OH, United States
}

Keywords: Ebola, sofosbuvir, RNA virus, RNA dependent RNA polymerase, triphosphate metabolite

\section{OPEN ACCESS}

Edited by:

Salvatore Salomone,

Università degli Studi di Catania, Italy

Reviewed by:

Takashi Irie,

Hiroshima University, Japan

${ }^{*}$ Correspondence:

Charles R. Ashby Jr. cnsratdoc@optonline.net.

Specialty section:

This article was submitted to Experimental Pharmacology and Drug

Discovery,

a section of the journal

Frontiers in Pharmacology

Received: 18 June 2018

Accepted: 19 September 2018 Published: 10 October 2018

Citation:

Reznik SE, Tiwari AK and Ashby CR Jr (2018) Sofosbuvir: A Potential Treatment for Ebola.

Front. Pharmacol. 9:1139. doi: 10.3389/fphar.2018.01139
There are currently no effective licensed vaccines or proven drugs available for the treatment of Ebola, which causes one of the deadliest viral diseases. The urgent need to identify novel and effective pharmacologic approaches to treat Ebola infections is underlined by recent reports of new Ebola outbreaks from the Centers for Disease Control and Prevention (CDC), including the outbreak in the Eastern Democratic Republic of the Congo on August 1, 2018. Here we present an argument as to why sofosbuvir, presently used to treat chronic hepatitis $\mathrm{C}$ virus (HCV) infections, could be an ideal candidate for the treatment of Ebola infection.

Ebola is a negative-sensed, non-segmented RNA virus and as with all RNA viruses, including $\mathrm{HCV}$, uses the enzyme RNA-dependent RNA polymerase (RdRp, transcribed from the Ebola $\mathrm{L}$ gene), along with other proteins, to replicate, maintain, and express its RNA genome (Trunschke et al., 2013) by (1) binding to an appropriate complementary $5^{\prime}$-nucleotide triphosphate and converting it to a $5^{\prime}$-nucleotide monophosphate and (2) catalyzing the interaction between the NMP and a $3^{\prime}$-hydroxyl-ribonucleotide to form a $5^{\prime}-3^{\prime}$ phosphodiester bond (McDonald, 2013). This process elongates the daughter RNA strand. The three dimensional structure of RdRp from RNA viruses resembles that of a cupped right hand and contains a finger, thumb, and palm domain (McDonald, 2013). Further, the palm domain of all viral RdRps, which mediate catalysis for RNA polymerization, have motifs A-E, and motifs A-C are the most conserved (Jácome et al., 2015). The predicted three-dimensional structure of the Ebola RdRp is similar to other viral RdRp (including $\mathrm{HCV}$ ) as it has the homologous and highly conserved catalytic domains A-C in the palm (Jácome et al., 2015). Furthermore, like all other viral RdRp, Ebola RdRp utilizes 2 divalent metal ions to catalyze ribonucleotide polymerization (Jácome et al., 2015). The Ebola RdRp is an excellent pharmacological target as (1) its inhibition will decrease viral replication; (2) there is no similar protein target in human cells.

Sofosbuvir ( $400 \mathrm{mg}$ p.o. once daily for 12 or 24 weeks), in combination with other drugs, is used to treat chronic HCV infections (Keating, 2014). Sofosbuvir is a uridine analog nucleotide phosphoramidate prodrug that is ultimately bio-transformed in hepatocytes to a triphosphate metabolite (GS-461203) that inhibits HCV RdRp (also known as the NS5B protein)-catalyzed RNA synthesis, thereby inhibiting viral replication and transcription (Sofia, 2013). GS-461203 has a halflife of $38 \mathrm{~h}$ in human hepatocytes (Summers et al., 2014), and reaches concentrations that exceed the $\mathrm{EC}_{50}$ for Ebola RdRp, ensuring that it will produce sustained antiviral action. Interestingly, the triphosphate metabolite of the adenine analog BCX4430 inhibits HCV RdRp in vitro and protects mice against Ebola (Warren et al., 2014). Furthermore, the triphosphate metabolite of the nucleoside analog favipiravir (T-705) inhibits RdRp activity of numerous RNA viruses and protects mice against Ebola-induced mortality 6 days after infection (Oestereich et al., 2014). However, there was no statistically significant decrease in the mortality rate in patients from Guinea (Sissoko et al., 2016) and Sierra Leone (Bai et al., 2016) during the 2013-2016 Ebola epidemic patients that received favipiravir. Furthermore, there were patients who were evacuated from 
West Africa to Europe who received favipiravir, but its effect on patient survival could not be determined as all of these patients received advanced supportive care and the majority were given additional experimental treatment (Mora-Rillo et al., 2015; Schibler et al., 2015; Agrati et al., 2016). It should be noted that in the JIKI trial (single-arm, proof-of-concept design), the plasma concentrations of favipiravir did not reach the target levels set prior to the trial (Nguyen et al., 2017).

The i.v. administration of $10 \mathrm{mg} / \mathrm{kg}$ of GS-5734 (once daily for 12 days), an adenine analog nucleotide phosphoramidate prodrug that is bio-transformed to an active nucleoside triphosphate compound, significantly decreased Ebola virus replication and protected $100 \%$ of rhesus monkeys against lethal Ebola virus (from 1995 outbreak in Kikwit, Zaire) infection (Warren et al., 2016). GS-5734 inhibits $\left(E_{50}=86 \mathrm{nM}\right.$ ) Ebola virus replication in human macrophages (Siegel et al., 2017). Furthermore, GS-5734 has completed phase I trials and is undergoing Phase II trials to determine its efficacy in Ebola survivors who have persistent viremia in semen (Bixler et al., 2017). The triphosphate metabolites of sofosbuvir (Sofia, 2013), BCX4430 (Warren et al., 2014), favipiravir (Bai et al., 2016), and GS-5734 (Warren et al., 2016) are incorporated into the viral RNA template and inhibit RdRp activity via chain termination. Thus, certain compounds that are active against HCV RdRp may also be efficacious in inhibiting Ebola RdRp.

We propose that prior to clinical trials, sofosbuvir's efficacy be tested in vitro against human macrophages and Huh-7 cells infected with the Ebola Makona variant. If these results indicate that sofosbuvir is efficacious, we propose that its in vivo efficacy be determined in a non-human primate model of Ebola. If these results are positive, sofosbuvir's efficacy in humans could be determined by measuring Ebola virus RNA in the semen of males 18-65 years old $(n=40)$ who were identified as having had a PCR-confirmed Ebola diagnosis in a double-blind, randomized, placebo-controlled trial. A 400-mg dose of sofosbuvir would be administered orally once daily for 28 days to individuals in the test group and a placebo tablet given to the control participants. The presence of Ebola RNA in all samples would be determined using real-time RT-PCR. Samples would be obtained once per week during treatment and once per month thereafter. The presence of infectious Ebola virus in the semen samples would be determined in severe combined immunodeficient mice as previously described (Sissoko et al., 2017). During treatment, all patients would be monitored for potential adverse effects via interviews and by obtaining blood samples. Following the treatment period, samples would be collected once a month for at least 13 months. All collected seminal fluid samples in the trial proposed here would be tested for the presence of Ebola virus RNA, and the clearance rate of the virus over 13 months in the two groups would be compared. If there is a statistically significant increase in viral clearance in the treated group as compared to the placebo control group, the clinical efficacy of sofosbuvir in humans could be determined by identifying individuals 18 years of age or older in a future epidemic who have laboratory-confirmed Ebola infection for a double-blind, randomized, placebo-controlled trial. Recent data suggest that the mortality rate from Ebola is about $50 \%$. Using this conservative estimate, and the modest prediction that sofosbuvir would decrease the mortality rate to $30 \%$, sample sizes of $n=$ 60 each for the control and drug groups would be sufficient to detect a statistically significant effect $(p<0.05)$. A 400-mg dose of sofosbuvir would be administered orally once daily for 28 days to individuals in the test group and a placebo tablet given to the control participants. The main efficacy endpoint would be survival after 28 days. Also, all placebo and sofosbuvir patients would receive the best available supportive care. Since we do not know whether sofosbuvir will significantly lower the mortality rate, the use of placebo is justified. Finally, patients should be followed to determine their viral load and acquired resistance to sofosbuvir.

Clinical studies and post-marketing data suggest that sofosbuvir has a highly favorable safety profile (Keating and Vaidya, 2014). The most common adverse effects produced by sofosbuvir are headache, nausea, dizziness, fatigue and abdominal pain, and no dose-limiting toxicities have been reported (Keating and Vaidya, 2014). Nonetheless, sofosbuvir treatment will be discontinued if it elicits severe or problematic adverse effects or increases mortality.

Patients should be screened for $\mathrm{HCV}$ as using sofosbuvir alone for the treatment of HCV would not be considered optimal therapy. It is recommended that sofosbuvir should not be taken by women who are breastfeeding. Animal data indicate that sofosbuvir and its predominant circulating metabolite, GS331007, at doses far exceeding those used in HCV patients, does not produce carcinogenicity, mutagenicity, or an impairment of fertility (Product Information, Gilead Sciences, Inc., 2015). A recent in vitro study indicates that sofosbuvir, at high concentrations, does not have toxic effects on the following human cell lines: hepatic (Huh7, HepG2), prostate (PC-3), fibroblasts (MRC5), T cells (MT-4), bone marrow erythroid, and myeloid cells (Feng et al., 2016). Finally, sofosbuvir (>200 $\mu \mathrm{M})$ does not significantly inhibit (1) the activity of the mitochondrial DNA polymerases alpha, beta, and gamma or (2) mitochondrial protein synthesis and respiration in PC-3 cells (Feng et al., 2016).

Clinical data suggest that sofosbuvir's pharmacokinetic profile is highly suitable for the potentially diverse populations of patients presenting with Ebola infection (Kirby et al., 2015). Notably, sofosbuvir has a large volume of distribution (1271), thereby increasing the likelihood that sufficient concentrations will be present in reservoir areas (e.g., eyes, testes, CNS) that support active Ebola replication. Current guidelines indicate that sofosbuvir can be used in patients with severe liver impairment and mild or moderate [estimated glomerular filtration rate $(\mathrm{eGFR})>30 \mathrm{ml} / \mathrm{min}$ ] impairment of renal function (Kirby et al., 2015). However, 4 recent studies (Hundemer et al., 2015; Dumortier et al., 2016; Nazario et al., 2016; Singh et al., 2016) in patients with chronic HCV (total of 80 patients) have reported that sofosbuvir (in combination with other anti-HCV drugs) for 12 to 24 weeks was well tolerated at doses of $400 \mathrm{mg} /$ day, $400 \mathrm{mg}$ every other day or 3 times a week in the presence of end stage renal disease or in patients with eGFR $<30 \mathrm{ml} / \mathrm{min}$. Thus, the use of sofosbuvir could be considered in Ebola patients with severe renal impairment in the absence of alternatives and with careful monitoring. The absorption of sofosbuvir 
could be affected by vomiting or diarrhea and it cannot be given to patients who are unconscious or have the inability to swallow the tablets. These issues could be addressed by i.v. administration, but there is no i.v. formulation for sofosbuvir. Sofosbuvir could be given in a solution of sulfobutyletherbeta-cyclodextrin, although the pharmacokinetic profile of this formulation remains to be determined. Alternatively, the sofosbuvir tablet could be disintegrated into water, juice, or milk with spoon stirring and light press and given via a nasoduodenal tube, although the pharmacokinetics of this formulation is unknown (Li and Foisy, 2014). Currently, the efficacy and safety of sofosbuvir for pediatric patients has not been reported.

Sofosbuvir and its main metabolite are not known to be substrates for CYP450 drug metabolizing enzymes and do not induce or inhibit these enzymes (Kirby et al., 2015). Sofosbuvir is a substrate for the ABC transporters p-glycoprotein (ABCB1) and breast cancer resistant protein (BRCP or ABCG2 transporter) (Kirby et al., 2015). Overall, sofosbuvir has a low liability to elicit significant drug-drug interactions. Indeed, the number of clinically significant drug-drug interactions are minimal for sofosbuvir (it should not be co-administered with potent inducers of intestinal ABCB1 and/or ABCG2, carbamazepine, oxcarbazepine, phenytoin, phenobarbital tipranivir + ritonavir, rifampin, rifabutin, rifapentine, or amiodarone) (Product Information, Gilead Sciences, Inc., 2015). This is important as Ebola patients may be receiving many drugs as part of their treatment regimen and based on published data, sofosbuvir is highly unlikely to attenuate the efficacy and/or increase the toxicity of numerous other drugs used to treat concomitant infections.

The cost of sofosbuvir is a critical issue regarding its use for Ebola. The median nominal ex-factory cost of a 12-week regimen of sofosbuvir for treating $\mathrm{HCV}$, across 26 countries in

\section{REFERENCES}

Agrati, C., Castilletti, C., Casetti, R., Sacchi, A., Falasca, L., Turchi, F., et al. (2016). Longitudinal characterization of dysfunctional $\mathrm{T}$ cell activation during human acute Ebola infection. Cell Death Dis. 7:e2164. doi: 10.1038/cddis.2016.55

Bai, C. Q., Mu, J. S., Kargbo, D., Song, Y. B., Niu, W. K., Nie, W. M., et al. (2016). Clinical and virological characteristics of Ebola virus disease patients treated with favipiravir (T-705)-Sierra Leone, 2014. Clin. Infect. Dis. 63, 1288-1294. doi: $10.1093 /$ cid/ciw571

Bixler, S. L., Duplantier, A. J., and Bavari, S. (2017). Discovering drugs for the treatment of Ebola virus. Curr. Treat. Options Infect. Dis. 9, 299-317. doi: 10.1007/s40506-017-0130-z

Dumortier, J., Leroy, V., Duvoux, C., De Ledinghen, V., Francoz, C., HousselDebry, P., et al. (2016). Sofosbuvir-based treatment of hepatitis C with severe fibrosis (METAVIR F3/F4) after liver transplantation. Liver Transpl. 22, 1367-1378. doi: 10.1002/lt.24505

Feng, J. Y., Xu, Y., Barauskas, O., Perry, J. K., Ahmadyar, S., Stepan, G., et al. (2016). Role of mitochondrial RNA polymerase in the toxicity of nucleotide inhibitors of hepatitis C virus. Antimicrob. Agents Chemother. 60, 806-817. doi: 10.1128/AAC.01922-15

Hill, A., Khoo, S., Fortunak, J., Simmons, B., and Ford, N. (2014). Minimum costs for producing hepatitis $\mathrm{C}$ direct-acting antivirals for use in large-scale treatment access programs in developing countries. Clin. Infect. Dis. 58, 928-936. doi: 10.1093/cid/ciu012 the Organization for Economic Cooperation and Development (OECD), was \$42,017 (Iyengar et al., 2016). This aforementioned price range would make sofosbuvir unavailable as a potential treatment to most patients on a global level. However, the cost of generic sofosbuvir in India, ranges from $\$ 161$ to 312 for 28 tablets (Iyengar et al., 2016). Thus, a 4-week regimen of sosfobuvir, as proposed in this paper, would be projected to cost $\$ 161$ to 312. Furthermore, it has been estimated that based upon (1) the manufacturing cost of retroviral drugs with similar mechanisms of action and chemical structures and (2) treating a minimum of 1 million people (Hill et al., 2014), a 12-week regimen of sofosbuvir should cost $\$ 68-136$, or $\$ 23-45$ for 4 weeks.

In conclusion, we hypothesize that sofosbuvir, a highly safe and effective treatment for HCV, if given in a timely manner, would decrease Ebola-induced mortality by lowering viral load. There is currently no drug that has proven to be efficacious against Ebola virus in a clinical setting, including favipiravir, and the safety profile of sofosbuvir is already well known. If sofosbuvir treatment significantly reduces Ebola mortality, its efficacy should be tested for prophylaxis and for post exposure prophylaxis. Also, its use could be considered in patients diagnosed with post-Ebola syndrome, given that one of the potential causes could be viral reservoirs.

\section{AUTHOR CONTRIBUTIONS}

SR, CA, and AT discussed and agreed on the presented opinion. CA wrote the draft and SR and AT contributed to a portion of the draft. CA, AT, and SR proofed the article.

\section{FUNDING}

AT will pay the cost of this article.
Hundemer, G. L., Sise, M. E., Wisocky, J., Ufere, N., Friedman, L. S., Corey, K. E., et al. (2015). Use of sofosbuvir-based direct-acting antiviral therapy for hepatitis $\mathrm{C}$ viral infection in patients with severe renal insufficiency. Infect. Dis. 47, 924-929. doi: 10.3109/23744235.2015.10 78908

Iyengar, S., Tay-Teo, K., Vogler, S., Beyer, P., Wiktor, S., De Joncheere, K., et al. (2016). Prices, costs, and affordability of new medicines for hepatitis C in 30 countries: an economic analysis. PLoS Med. 13:e1002032. doi: 10.1371/journal.pmed.1002032

Jácome, R., Becerra, A., Ponce de León, S., and Lazcano, A. (2015). Structural analysis of monomeric RNA-dependent polymerases: evolutionary and therapeutic implications. PLOS ONE 10:e0139001. doi: 10.1371/journal.pone.0139001

Keating, G. M. (2014). Sofosbuvir: a review of its use in patients with chronic hepatitis C. Drugs 74, 1127-1146. doi: 10.1007/s40265-014-0247-Z

Keating, G. M., and Vaidya, A. (2014). Sofosbuvir: first global approval. Drugs 74, 273-282. doi: 10.1007/s40265-014-0179-7

Kirby, B. J., Symonds, W. T., Kearney, B. P., and Mathias, A. A. (2015). Pharmacokinetic, pharmacodynamic, and drug-interaction profile of the hepatitis C virus NS5B polymerase inhibitor sofosbuvir. Clin. Pharmacokinet. 54, 677-690. doi: 10.1007/s40262-015-0261-7

Li, E. H., and Foisy, M. M. (2014). Antiretroviral and medication errors in hospitalized HIV-positive patients. Ann. Pharmacother. 48, 998-1010. doi: $10.1177 / 1060028014534195$ 
McDonald, S. M. (2013). RNA synthetic mechanisms employed by diverse families of RNA viruses. Wiley Interdiscip. Rev. RNA 4, 351-367. doi: 10.1002/wrna .1164

Mora-Rillo, M., Arsuaga, M., Ramirez-Olivencia, G., de la Calle, F., Borobia, A. M., Sanchez-Seco, P., et al. (2015). Acute respiratory distress syndrome after convalescent plasma use: treatment of a patients with Ebola virus disease contracted in Madrid, Spain. Lancet Respir. Med. 3, 554-562. doi: 10.1016/S2213-2600(15)00180-0

Nazario, H. E., Ndungu, M., and Modi, A. A. (2016). Sofosbuvir and simeprevir in hepatitis $C$ genotype 1-patients with end-stage renal disease on haemodialysis or GFR $<30 \mathrm{ml} / \mathrm{min}$. Liver Int. 36, 798-801. doi: 10.1111/liv. 13025

Nguyen, T. H., Guedj, J., Anglaret, X., Laouenan, C., Madelain, V., Taburet, A. M., et al. (2017). Favipiravir pharmacokinetics in Ebola-infected patients of the JIKI trials reveals lower concentrations than target. PLoS Negl. Trop. Dis. 11:e0005389. doi: 10.1371/journal.pntd.0005389

Oestereich, L., Lüdtke, A., Wurr, S., Rieger, T., Muñoz-Fontela, C., and Günther, S. (2014). Successful treatment of advanced Ebola virus infection with T-705 (favipiravir) in a small animal model. Antiviral Res. 105, 17-21. doi: 10.1016/j.antiviral.2014.02.014

Product Information, Gilead Sciences, Inc. (2015). Sovaldi (sofosbuvir). Foster City, CA: Gilead Science, Inc.

Schibler, M., Vetter, P., Cherpillod, P., Petty, T. J., Cordey, S., Vieille, G., et al. (2015). Clinical features and viral kinetics in a rapidly cured patient with Ebola virus disease: a case report. Lancet Infect. Dis. 15, 1034-1040. doi: 10.1016/S1473-3099(15)00229-7

Siegel, D., Hui, H. C., Doerffler, E., Clarke, M. O., Chun, K., Zhang, L., et al. (2017). Discovery and synthesis of a phosphoramidate prodrug of a Pyrrolo[2,1-f][triazin-4-amino] Adenine C-Nucleoside (GS-5734) for the treatment of Ebola and emerging viruses. J. Med. Chem. 60, 1648-1661. doi: 10.1021/acs.jmedchem.6b 01594

Singh, T., Guirguis, J., Anthony, S., Rivas, J., Hanouneh, I. A., and Alkhouri, N. (2016). Sofosbuvir-based treatment is safe and effective in patients with chronic hepatitis $\mathrm{C}$ infection and end stage renal disease: a case series. Liver Int. 36, 802-806. doi: 10.1111/liv.13078
Sissoko, D., Duraffour, S., Kerber, R., Kolie, J. S., Beavogui, A. H., Camara, A. M., et al. (2017). Persistence and clearance of Ebola virus RNA from seminal fluid of Ebola virus disease survivors: a longitudinal analysis and modelling study. Lancet Glob Health 5, e80-e88. doi: 10.1016/S2214-109X(16)30243-1

Sissoko, D., Laouenan, C., Folkesson, E., M'Lebing, A. B., Beavogui, A. H., Baize, S., et al. (2016). Experimental treatment with favipiravir for Ebola virus disease (the JIKI Trial): a historically, single-arm proof-of-concept trial in Guinea. PLoS Med. 13:e1001967. doi: 10.1371/journal.pmed.1001967

Sofia, M. J. (2013). Nucleotide prodrugs for the treatment of HCV infection. Adv. Pharmacol. 67, 39-73. doi: 10.1016/B978-0-12-405880-4.00002-0

Summers, B. B., Beavers, J. W., and Klibanov, O. M. (2014). Sofosbuvir, a novel nucleotide analogue inhibitor used for the treatment of hepatitis $\mathrm{C}$ virus. $J$. Pharm. Pharmacol. 66, 1653-1666. doi: 10.1111/jphp.12294

Trunschke, M., Conrad, D., Enterlein, S., Olejnik, J., Brauburger, K., and Mühlberger, E. (2013). The L-VP35 and L-L interaction domains reside in the amino terminus of the Ebola virus $\mathrm{L}$ protein and are potential targets for antivirals. Virology 441, 135-145. doi: 10.1016/j.virol.2013.03.013

Warren, T. K., Jordan, R., Lo, M. K., Ray, A. S., Mackman, R. L., Soloveva, V., et al. (2016). Therapeutic efficacy of the small molecule GS-5734 against Ebola virus in rhesus monkeys. Nature 531, 381-385. doi: 10.1038/nature17180

Warren, T. K., Wells, J., Panchal, R. G., Stuthman, K. S., Garza, N. L., Van Tongeren, S. A., et al. (2014). Protection against filovirus diseases by a novel broad-spectrum nucleoside analogue BCX4430. Nature 508, 402-405. doi: 10.1038 /nature13027

Conflict of Interest Statement: The authors declare that the research was conducted in the absence of any commercial or financial relationships that could be construed as a potential conflict of interest.

Copyright (c) 2018 Reznik, Tiwari and Ashby. This is an open-access article distributed under the terms of the Creative Commons Attribution License (CC BY). The use, distribution or reproduction in other forums is permitted, provided the original author(s) and the copyright owner(s) are credited and that the original publication in this journal is cited, in accordance with accepted academic practice. No use, distribution or reproduction is permitted which does not comply with these terms. 